\title{
Track Targets Identification in Store Separation Tests using Digital Image Processing Techniques
}

\author{
A. André Yoshimi Kusumoto ${ }^{1,2}$, B. MSc. Luiz Eduardo Guarino de Vasconcelos ${ }^{1,2}$, C. Dr. Nelson Paiva \\ Oliveira Leite ${ }^{2}$, D. Dr. Rogério Pirk ${ }^{1}$ \\ ${ }^{1}$ Instituto Tecnológico de Aeronáutica (ITA) \\ Pça Mal. Eduardo Gomes $n^{\circ}$ 50, Vila das Acácias, São José dos Campos, SP \\ 12.228-901 - Brazil \\ A cti-p@ipev.cta.br; B cti-i@ipev.cta.br; D pirk@iae.cta.br \\ 2Instituto de Pesquisas e Ensaios em Voo (IPEV) \\ Pça Mal. Eduardo Gomes n 50, Vila das Acácias, São José dos Campos, SP \\ 12.228-901 - Brazil \\ Cepd@ipev.cta.br
}

\begin{abstract}
The digital image processing techniques, specifically the area of Computer Vision, has long been used lately in applications with human-machine interaction in surveillance and military applications, such as for the identification of people in an environment monitored by cameras or extraction of information from different positions in images of an aircraft Head-Up Display (HUD). The same techniques can be used in other situations such as in flight tests. In Store Separation Tests, the data analysis occurs post flight using the commercial off-the-shelf applications, but still depends of the user accuracy which indicates where the track targets are. This process can be time consuming and unproductive. The agility to achieve the analysis results can be improved with the use of computer vision techniques. The Instituto de Pesquisas e Ensaios em Voo (IPEV) together with the Instituto Tecnológico de Aeronáutica (ITA) is currently developing an application that identify and tracks in real-time several reference points fixed in the store surface. This paper presents the study and application of techniques of digital image processing for identifying position of reference points in sequential images, the software implements algorithms that uses techniques to extract their features and Haar-like cascade and Local Binary Patterns (LBP) classifiers. The preliminary results were considered satisfactory.
\end{abstract}

Keywords: flight test, store separation, track targets, cascade classifiers, digital image processing

\section{Introduction}

Flight test is an indispensable phase for aeronautic product development, certification and qualification. Typically, the execution of a Flight Test Campaign requires the execution of several test flights to gather the required information. For each flight, all related aspects should be well defined (e.g. Risk Level) and planned (e.g. Flight Profile) to: a) Achieve a particular purpose; b) Ensure the test success; and c) Avoid incidents that could result in material (e.g. aircrafts and/or weapons) and human (e.g. test crew) losses and/or damages. Depending on the test, different parameter set should be acquired by the airborne Flight Test Instrumentation System (FTI).

In Store Separation Tests the minimum parameter set requires the acquisition of both test bed and released store Time-Space Position Information (TSPI) for the determination if the store trajectory is safe or not while non-linear and unpredicted aerodynamics couplings between the released store and the aircraft structure (e.g. Wings and stabilizers) could lead to a catastrophic collision condition. Furthermore for ballistic stores the effects of non-uniform phase are significant and important for computing the estimated release or impact points.

For external store TSPI determination the FTI set uses $\mathrm{Hi}$-Speed $\mathrm{Hi}$-Resolution video cameras (e.g 720i and up to 400 frames/s) for raw data gathering (i.e. Video Frames). The TSPI data extraction process, called photogrammetry, can be performed by computational resources that analyze captured videos frames. Such analysis requires massive data processing resources to identify multiple 
reference points (i.e. track targets) marked on the store surface and in sequence to determine its $3-D$ coordinates in a reference frame. Several commercial off-the-shelf applications (e.g. Trackeye) can perform such function as post mission data analysis. Furthermore, the introduction of real-time photogrammetric application can assist engineers during test flight to determine if a given test point was safe or not. Therefore the usage of such facility can improve test efficiency because the test crew will be able to execute multiple release test points in a single flight. The benefit of such tool includes: a) Better usage of resources (i.e. Flight hours); b) Workload minimization; and c) Time and cost reduction.

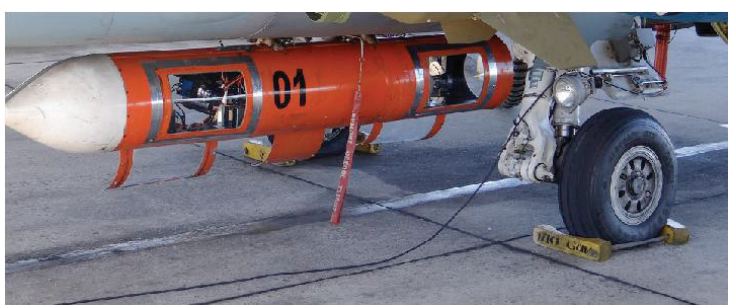

Figure 1 - Photogrammetric pod used for external stores release FTC[9]

Then, using techniques of digital image processing, the Instituto de Pesquisas e Ensaios em Voo (IPEV) together with the Instituto Tecnológico de Aeronáutica (ITA), under financial support of Financiadora de Estudos e Projetos (FINEP), under project FINEP-FEV, ref.: 01.12 .0518 .00 and administrative support of Fundação de Ciência, Aplicações e Tecnologia Espaciais (FUNCATE) is currently developing an airborne real time tracking system for stores separation FTC. The core of the system is an application that identify and tracks in real-time several reference points fixed in the store surface. This application analyzes the images generated by $\mathrm{Hi}$-Speed $\mathrm{Hi}-$ Resolution video camera installed on the aircraft and uses the Open Source Computer Vision (OpenCV).

In this initial phase, for identifying position of reference points in sequential images, the software implements algorithms that uses techniques to extract their features (e.g. area, contours, centroid, orientation, perimeter, equivalent diameter) and a cascade classifier. Videos of store separation tests conducted by EFEV (i.e. Brazilian Flight Test School) 2013 class students were used to evaluate the software and the results were considered satisfactory.

\section{Object Recognition}

\section{Techniques}

There are several techniques in computer vision for objects recognition as SIFT[1], SURF[2], Haar-like cascade[3] and Local Binary Patterns (LBP) [4]. Both Haar as LBP, has been used primarily for face recognition, but can also be used for recognizing other objects. The great majority of these techniques is based on the extraction of target object descriptors. Descriptors are characteristics of a particular region or object that will be used as a pattern recognition. These attributes are used in different ways to identify the object.

The Haar cascade detector, proposed by [3], uses Haar-like attributes for object detection. The attributes uses the difference of contrasts values between adjacent pixels. Three rectangular features are used for determining the specific object features: the attribute value of two-rectangles is the difference between the sum of the pixels within the rectangles, threerectangles attribute value is the sum of two outside rectangles subtracted from the sum of the center rectangle and four-rectangles attribute value is the difference between diagonal pairs.

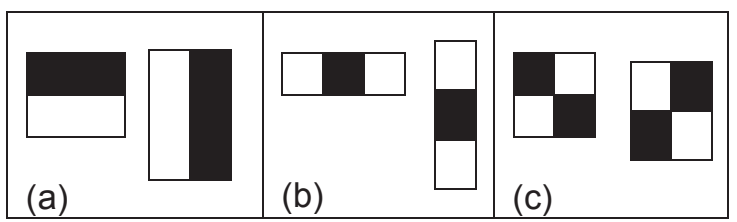

Figure 2 - Two-rectangles (a), three-rectangles (b) and four-rectangles features (c)

Using these three bases and the positive and negative samples set, the machine learning algorithm AdaBoost (i.e. Adaptive Boosting) is used to select an attributes set and train the classifier [3]. At each training stage the threshold values are adjusted to minimize the false negatives.

The LBP method is simpler and faster than Haar to extract the attributes. The LBP attributes are extracted from the division of the source image into small sub-windows of the same size, usually $3 \times 3$ pixels [5]. In each sub-window, a value is extracted from the threshold value of its pixels from the value of the central pixel threshold. Then binary values for each pixel, with the exception of the central pixel are assigned. These values are used to obtain a final value for that attribute used as subwindow. Other authors have extended this method and become more robust and faster 
$[4,6]$. It can also be used with the AdaBoost learning algorithm.

A Haar or LBP classifier can be trained with a hundreds positives samples (i.e. images where the object is present) and other a hundreds of negatives samples (i.e. images where the object is not present). A file, typically an eXtensible Markup Language (XML) format, containing the results of the training is generated from the training. This file is used to perform object recognition in a sequence of images or video.

\section{Track Targets}

\section{Identification Tool}

The application was developed using the OpenCV 2.4.8.0 library with the $\mathrm{C}++$ language interface. The operating system used is Linux Ubuntu 13.10. The OpenCV [7] library is a multiplatform and an open source framework for developing applications in the area of Computer Vision. Provides image manipulation and video tools, and includes several hundreds of computer vision algorithms. To implement the track targets identification tool was necessary perform two-steps: training process and track targets recognition process. The OpenCV provides several tools and an extensive online documentation to help the developer. The training process involves the following steps:

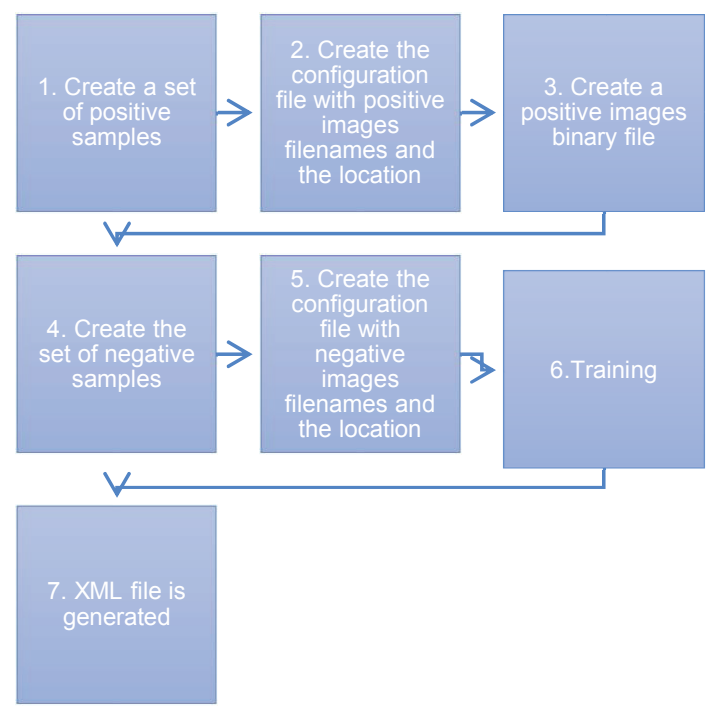

Figure 3 - The 7 steps of training process

The composition of positive samples set is an important step for the application. Region of interest (ROI) of frames from the video source and some images of the target from different sources were used. The set of positive samples (1) used in training has 240 images, all with $20 \times 20$ pixels. All positive images must have the same dimension. Then, create a configuration file of positive samples (2) which is a text file with the filenames and the location of the positive images. A binary file that contains images is created to prepare a training dataset of positive samples (3).

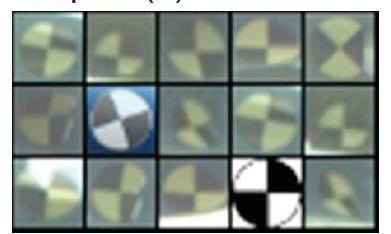

Figure 4 - Positive samples with 20x20 pixels.

Arbitrary images that do not contain the target were used to compose the database of negative samples. A set of 500 negative images, also called background samples, was generated (4). The images do not need to have the same dimension. The configuration file of negative samples (5) is generated the same format as the positive samples.

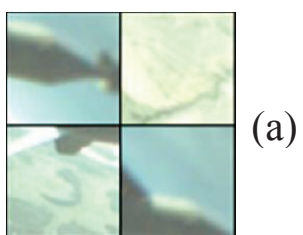

(b)

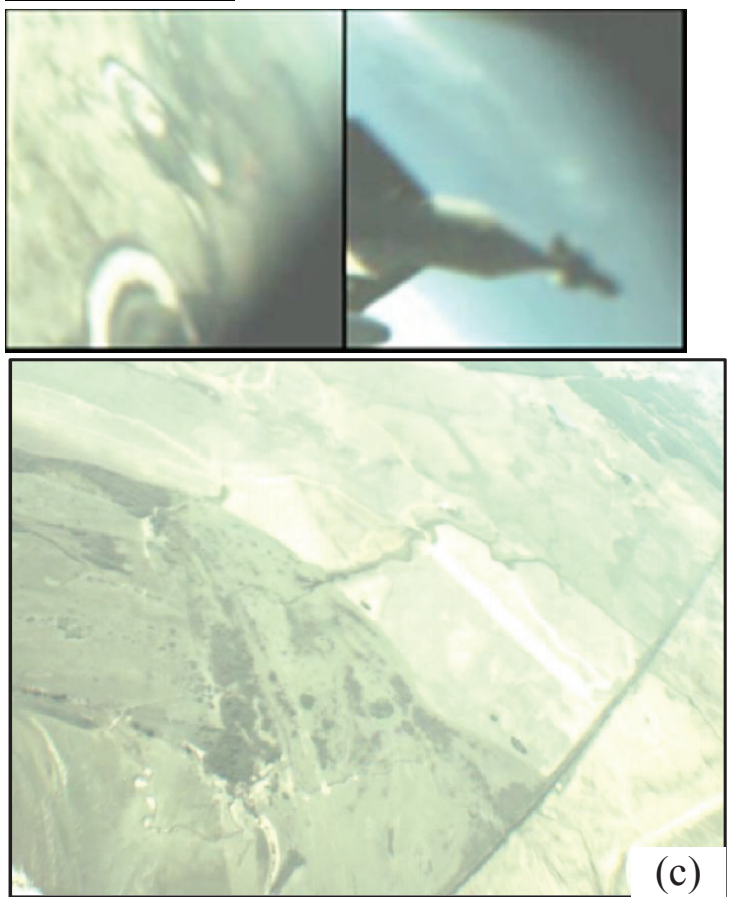

Figure 5 - Negatives samples with 53x53 pixels (a), $125 \times 125$ pixels (b) and $640 \times 480$ pixels (c)

The training (6) was executed in a notebook Dell Inspiron 14z, Intel ${ }^{\circ}$ Core $^{\mathrm{TM}}$ i7-3537U CPU @ $2.00 \mathrm{GHz} \times 4$, 8GB DDR3, 500GB 5400 RPM SATA HDD and 32GB mSATA SSD. The operating system used was Ubuntu 13.10. It is possible to choose the option of using the Haar or LBP attributes. At the end, the XML file is 
generated (7) containing the information of the trained classifier.

In recognition phase, after the load of cascade.xml file, each frame video captured by the application is processed. First, the frame is converted in a grayscale image and then, its histogram is equalized so that the brightness is normalized and contrast is improved. The detectMultiScale method was used, available in OpenCV CascadeClassifier class, to do the track target identification from image. The method save all the possible recognized objects into a vector data structure.

\section{Experiment Results}

The experiments were accomplished using videos from store separation tests, provided by EFEV. The analyzed video had high resolution, i.g. $1152 \times 1320$ pixels, and 5 frames per second sample rate. In this case, the low rate did not affect the results, due to the purpose was to analyze a single video frame for the track target identification. In tracking targets process, the sample rate is an important parameter to be considered, because the analysis is made frame by frame. The process works when the frame is loaded, an initial window is defined, and the tracking calculations is realized to predict the next localization, load the next frame, do the adjustment and show the results.

As the OpenCV provides the possibility of using the two methods during training, was set up the same amount of memory to compare the performance during the training process between the Haar and LBP. The precalcldxBufSize parameter is used to allocate more memory for the process. This parameter was set in $256 \mathrm{MB}$. The classifier was trained with 20 stages and minimum hit rate (i.e. minHitRate) was set in 0.999 per stage. The primary difference verified was the time length during the training process. The Haar-like process training took 1 hour, 18 minutes and 9 seconds. Using LBP, the process took 34 minutes and 17 seconds.

After load the XML file with the classifiers, the detectMultiScale is called. This method detects the objects in different sizes and return a rectangle vector. The minSize parameter that sets the size of minor rectangles to be recognized was defined in $20 \times 20$ pixels and the maxSize parameter that sets the size of major rectangles was defined in $80 \times 80$ pixels. Any value less than minSize or more than maxSize was disregarded.

The red rectangles shows the track targets identified. In addition to the targets in the store, it was decided also to keep the targets identification at wing pylon, which can be later used for calculations reference.

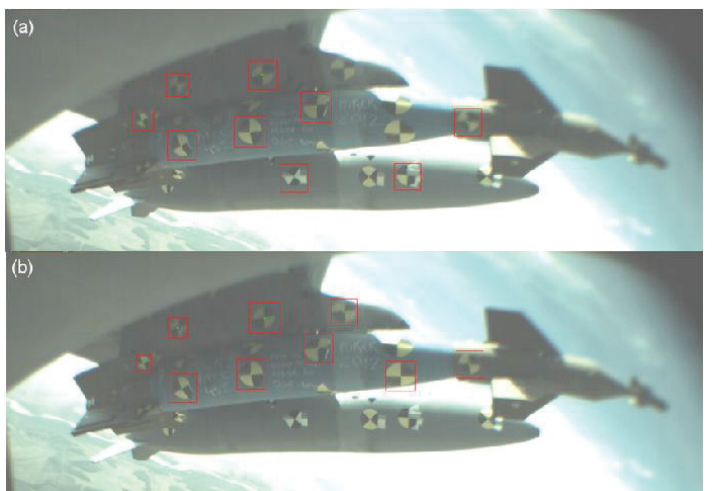

Figure 6 - Track targets identification results using the Haar (a) and LBP (b) attributes - frame 1.

The results were similar with the same samples set. In frame 1, the LBP method was able to identify all the targets in the store. In frame 74 , the Haar got a better result.

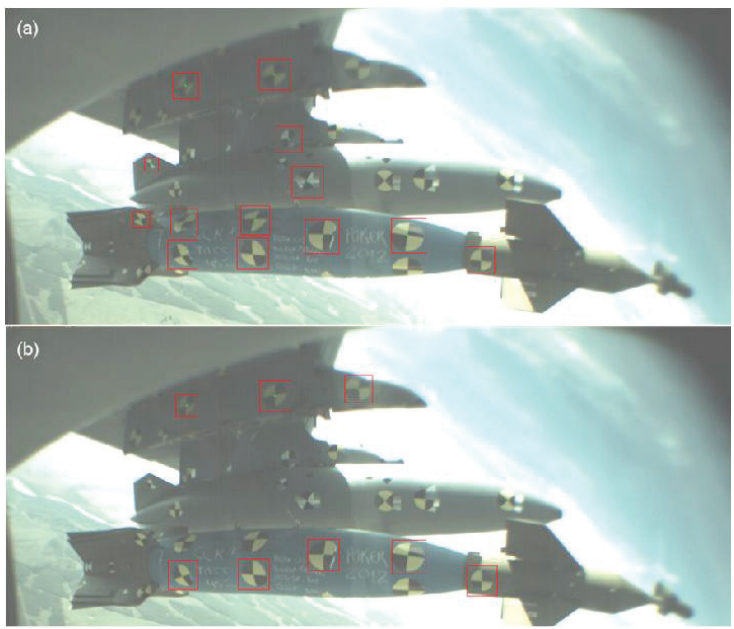

Figure 7 - Track targets identification results using the Haar (a) and LBP (b) attributes - frame 74

The accuracy achieved by algorithms for a total of 618 tracks targets is presented in Table 1.

Table 1 - Results after track targets identification process

\begin{tabular}{|l|c|c|c|}
\hline Type & $\begin{array}{c}\text { Identified } \\
\text { targets }\end{array}$ & $\begin{array}{c}\text { False } \\
\text { Positives }\end{array}$ & $\begin{array}{c}\text { False } \\
\text { Negatives }\end{array}$ \\
\hline Haar & $92,23 \%$ & 322 & $7,77 \%$ \\
\hline LBP & $87,86 \%$ & 20 & $12,14 \%$ \\
\hline
\end{tabular}

Compared with the LBP method, the Haar method achieved the best result in the identification of targets and the minor rate of false negatives. On the other hand, showed a high rate of false positives, more than 16 times compared with LBP. This cannot be considered a problem, it is possible to treat the false positives by performing a confirmation of each 
object found, improving the accuracy of the result.

\section{Conclusions}

With this study, the techniques used for the identification of targets presented satisfactory results and new applications can be developed for testing the separation of different store. With some refinement of the algorithm, the presented results can be even better, i.e. increasing the number of negative samples to avoid false positives occurring. Any of the techniques of extraction of features used (e.g. Haar-like or LBP) showed good results and can be used as a starting point for use in new applications. As future work and from the targets identified, the position of each target can be passed as input parameter to another application and perform the track targets tracking of these objects in a video.

\section{Acknowledgement}

We wish to thank the unconditional support given by the Instituto de Pesquisas e Ensaios em Voo (IPEV) and Instituto Tecnológico de Aeronáutica (ITA). Also we like to thank FINEP under agreement 01.12.0518.00 that funded the development of this tool and the presentation trip.

\section{References}

[1] LOWE, D. G. Distinctive Image Features from Scale-Invariant Keypoints. International Journal of Computer Visio, 2004.

[2] BAY, H., TUYTELAARS, T.,VANGOOL L., Surf: Speeded up robust features, European Conference on Computer Vision, 2006.

[3] VIOLA, P.; JONES, M. Rapid object detection using a boosted cascade of simple features, IEEE Computer Society Conference on Computer Vision and Pattern Recognition. CVPR/USA, Los Alamitos: CVPR/USA 2001.

[4] LIAO, S.; ZHU, X.; LEI, Z.; ZHANG, L.; LI, S. Z. Learning Multi-scale Block Local Binary Patterns for Face Recognition. International Conference on Biometrics (ICB), 2007.

[5] OJALA, T., PIETIKAINEN, M., HARWOOD, D. A comparative study of texture measures with classification based on feature distributions. Pattern Recognition (1996)

[6] AHONEN, T., HADID, A., PIETIKAINEN, M Face recognition with local binary patterns. Proceedings of the European Conference on Computer Vision (ECCV), 2004.
[7] OPENCV. OpenCV 2.4.8.0 documentation [online]. Available at: http://docs.opencv.org. (Accessed 20 Apr. 2014).

[8] WILSON, P. I.; FERNANDEZ, J., Facial feature detection using Haar classifiers. JCSC, 2006.

[9] LEITE, N. P. O.; VASCONCELOS, L. E. G.; KUSUMOTO, A. Y. Fast On-Board Tracking System for External Stores Separation, Proceedings of the 49th Annual International Telemetering Conference ITC/USA 2013, Las Vegas: ITC/USA 2013, pp. 1088 -1097, ISSN 1546-2188.

[10] VASCONCELOS, L. E. G.; KUSUMOTO, A. Y.; LEITE, N. P. O. Using Image Processing and Pattern Recognition in Images From Head-Up Display, Proceedings of the 49th Annual International Telemetering Conference ITC/USA 2013, Las Vegas: ITC/USA 2013, pp. 1088 1097, ISSN 1546-2188.

[11] GONZALEZ, R. C.; WOODS R. E., Digital Image Processing - 3rd Edition, Prentice Hall. 